\title{
In vitro evaluation of a bone morphogenetic protein-2 nanometer hydroxyapatite collagen scaffold for bone regeneration
}

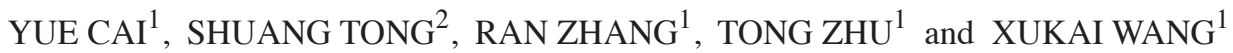 \\ ${ }^{1}$ Department of Oral and Maxillofacial Surgery, School of Stomatology, China Medical University, Liaoning Institute of \\ Dental Research; ${ }^{2}$ Department of Plastic Surgery, The First Affiliated Hospital of China Medical University, \\ Shenyang, Liaoning 110002, P.R. China
}

Received July 26, 2017; Accepted November 10, 2017

DOI: $10.3892 / \mathrm{mmr} .2018 .8579$

\begin{abstract}
Scaffold fabrication and biocompatibility are crucial for successful bone tissue engineering. Nanometer hydroxyapatite (nHAP) combined with collagen (COL) is frequently utilized as a suitable osseous scaffold material. Furthermore, growth factors, including bone morphogenetic protein-2 (BMP-2), are used to enhance the scaffold properties. The present study used blending and freeze-drying methods to develop a BMP-2-nHAP-COL scaffold. An ELISA was performed todetermine the BMP-2 releaserate from the scaffold. Flow cytometry was used to identify rat bone marrow-derived mesenchymal stem cells (BMSCs) prior to their combination with the scaffold. Scanning electron microscopy was used to observe the scaffold structure and BMSC morphology following seeding onto the scaffold. BMSCs were also used to assess the biological compatibility of the scaffold in vitro. BMP-2-nHAP-COL and nHAP-COL scaffolds were assessed alongside the appropriate control groups. Cells were counted to determine early cell adhesion. Cell Counting kit- 8 and alkaline phosphatase assays were used to detect cell proliferation and differentiation, respectively. Gross morphology confirmed that the BMP-2-nHAP-COL scaffold microstructure conformed to the optimal characteristics of a bone tissue engineering scaffold. Furthermore, the BMP-2-nHAP-COL scaffold exhibited no biological toxicity and was demonstrated to promote BMSC adhesion, proliferation and differentiation. The BMP-2-nHAP-COL scaffold had good biocompatibility in vitro, and may therefore be modified further to construct an optimized scaffold for future bone tissue engineering.
\end{abstract}

Correspondence to: Professor Xukai Wang, Department of Oral and Maxillofacial Surgery, School of Stomatology, China Medical University, Liaoning Institute of Dental Research, 117 Nan Jing North Street, Heping, Shenyang, Liaoning 110002, P.R. China E-mail: wangxukai757892@sina.com.cn

Key words: bone tissue engineering, bone marrow-derived mesenchymal stem cells, nanometer hydroxyapatite, collagen, biocompatibility, bone morphogenetic protein 2

\section{Introduction}

Tissue engineering has the potential to treat millions of patients living with debilitating diseases (1). The requirement for effective bone repair therapy arises from conditions including congenital malformation, trauma, tumor resection and skeletal disease (2). Typically, bone tissue engineering consists of harvesting cells from a patient, expanding them in vitro and culturing them into a biomaterial, additionally termed a scaffold. This functions as a structural framework to facilitate cell attachment, proliferation and differentiation into a controlled phenotype (3). Scaffold design is key to effective tissue engineering. A scaffold should provide an ideal microenvironment to promote cell and tissue growth. At a minimum, the scaffold should have adequate mechanical stability to withstand cellular contractile forces, high porosity with interconnected pores to facilitate nutrient delivery and remove metabolic waste, and must be biocompatible to promote tissue formation and integration (4-9). Growth factors are soluble proteins that stimulate cell growth and differentiation, which have emerged as broadly applicable tools for the induction of bone formation. Bone morphogenetic proteins (BMPs) are growth factors that are effective at orchestrating novel bone formation in humans by recapitulating the different stages of bone development (10). Hydroxyapatite (HAP)/collagen (COL) composites are typically used as bone substitute materials in dentistry and orthopedic surgery, for the regeneration of damaged hard tissue (11). Nanometer (n) HAP in particular has been widely used for tissue engineering scaffold construction (12-16). BMP-2 is the most extensively studied BMP in the context of osteogenesis and has been demonstrated to enhance bone formation (17-19). Vilquin and Rosset (20) reported that bone marrow-derived mesenchymal stem cells (BMSCs) lack immunogenicity, making them an ideal cell source for tissue engineering. Thus, BMSCs may be seeded into scaffolds and implanted into the body without producing a marked antigenic response. BMSCs have additionally been used to determine the biological toxicity of tissue-engineered scaffolds. In absence of biological toxicity in vitro, it is reasonable to hypothesize that the same scaffold will avoid antigen rejection following implantation.

In the present study, blending and freeze-drying methods were combined to construct the BMP-2-nHAP-COL scaffold. 
The scaffold properties were assessed to determine whether the requirements for bone tissue engineering had been met.

\section{Materials and methods}

BMP-2-nHAP-COL scaffold preparation. Acetic acid solution was diluted in deionized water to a concentration of $0.005 \mathrm{~mol} / \mathrm{l}$, and COL (10 mg; Shengyou Biotechnology Co., Ltd., Hangzhou, China) was subsequently added into $10 \mathrm{ml}$ acetic acid solution and stirred (JB-2A; Bante Instruments Ltd., Shanghai, China) for $50 \mathrm{~min}$. nHAP (10 mg; Emperor Nano Material Co., Ltd., Nanjing, China) was added to this solution and stirred overnight. The quality ratio was determined to be 1:1.5 (nHAP:COL). BMP-2 (PeproTech, Inc., Rocky Hill, NJ, USA) was dissolved using PeproTech protein solution to $10 \mathrm{ng} / \mu \mathrm{l}(21)$, and added to the scaffold solution prior to stirring for $50 \mathrm{~min}$ at $4^{\circ} \mathrm{C}$, resulting in a final BMP-2 concentration of $100 \mathrm{ng} / \mathrm{ml}$. The solution was added into a 24-well Teflon ${ }^{\mathrm{TM}}$ culture plate and frozen at $-20^{\circ} \mathrm{C}$ for $24 \mathrm{~h}$, and lyophilized at $-80^{\circ} \mathrm{C}$ for $48 \mathrm{~h}$ (VFD-2000; Boyikang Laboratory Instruments, Co., Ltd., Beijing, China) to form BMP-2-nHAP-COL scaffolds. Scaffold morphology and microstructure was observed by scanning electron microscopy (SEM).

Detection of BMP-2 release from BMP-2-nHAP-COL scaffolds. A total of three standard BMP-2-nHAP-COL scaffolds (BMP-2, $100 \mathrm{ng}$ ) were placed into a 24-well culture plate and 1X PBS buffer ( $1 \mathrm{ml}$; pH, 7.4) was added into the wells prior to sealing with a membrane. The plate was placed in an incubator at $37^{\circ} \mathrm{C}$, and PBS from the wells was collected at specific time points (1, 2 and 20 days). PBS samples were subsequently analyzed for BMP-2 content with a BMP-2 ELISA kit (cat. no. YD-H010379; Yuduo Biotechnology Company, Shanghai, China). The mean BMP-2 values were calculated to determine the cumulative release of BMP-2 and a release curve was subsequently drawn (22). The experiment was repeated three times.

Animals and ethics statement. A total of eight Sprague-Dawley rats (four male and four female, 4 weeks old, 300 g) were obtained from the Center for Experimental Animals at China Medical University (Shenyang, China; National Animal Use License no. SCXK-LN2011-0009). Animals were housed at a temperature of $20-26^{\circ} \mathrm{C}$ and a $12 \mathrm{~h}$ light/dark cycle with unlimited access to food and water. Animal use was approved by the Animal Use and Care Committee at China Medical University (protocol no. CMU62043006). All experiments were approved by the Animal Care and Use Committee at China Medical University, and complied with the National Institutes of Health (Bethesda, MD, USA) Guide for the Care and Use of Laboratory Animals. All efforts were made to minimize the number of animals used and their suffering.

Isolation, culture and passage of BMSCs. Rats were sacrificed with excess anesthesia and skin was sterilized with $75 \%$ ethanol. Under aseptic conditions, the femur and tibia were removed and placed in PBS solution. Following ultraviolet disinfection, the PBS liquid was drained and the bone was washed three times with PBS liquid containing penicillin and streptomycin. The bone marrow cavity was exposed and
$5.2 \mathrm{ml} \alpha$-Minimum Essential Medium ( $\alpha$ MEM; Hyclone; GE Healthcare Life Sciences, Logan, UT, USA) containing $10 \%$ fetal bovine serum (FBS; Hyclone; GE Healthcare Life Sciences), $1 \%$ penicillin and $1 \%$ streptomycin was drawn through until the majority of the bone marrow was flushed out. The cell suspension was washed and precipitated with PBS three times, and transferred to a sterile container. Cells were counted and seeded into culture dishes according to the required density. $\alpha \mathrm{MEM}$ containing $10 \% \mathrm{FBS}, 1 \%$ penicillin and $1 \%$ streptomycin was added, and cells were cultured at $37{ }^{\circ} \mathrm{C}$ in a $5 \% \mathrm{CO}_{2}$ incubator. The medium was changed every $24 \mathrm{~h}$. Following adherence to the culture dish, cells were digested using TrypLe Express enzyme (2.5-3.0 ml; Gibco; Thermo Fisher Scientific, Inc., Waltham, MA, USA) for $10 \mathrm{~min}$ at $37^{\circ} \mathrm{C}$ and $\alpha \mathrm{MEM}$ containing $10 \% \mathrm{FBS}$ was used to terminate the reaction. The cell suspension was centrifuged at $800 \mathrm{x} g$ for $5 \mathrm{~min}$, and cells were resuspended in fresh $\alpha \mathrm{MEM}$ containing 10\% FBS (both Hyclone; GE Healthcare Life Sciences) and seeded into new culture medium. Cell medium was changed daily and, once 80-90\% confluence was reached, cells were passaged at a ratio of 1:3. A light-inverted microscope (cat. no. CKX41; Olympus Corporation, Tokyo, Japan) was used to observe cell morphology.

BMSC identification. Third-generation BMSCs were used for identification. The medium was discarded and cells were washed three times with PBS. TrypLE Express was subsequently added to digest the cells at $37^{\circ} \mathrm{C}$ for $10 \mathrm{~min}$ and $\alpha \mathrm{MEM}$ (Hyclone; GE Healthcare Life Sciences) containing 10\% FBS was used to terminate the reaction. The cell suspension was collected and centrifuged at $500 \mathrm{x}$ g at $4^{\circ} \mathrm{C}$ for $5 \mathrm{~min}$. Cells were subsequently counted and adjusted to $10^{6}$ cells $/ 100 \mu \mathrm{l}$, and blocked using PBS containing $10 \%$ bovine serum albumin (concentration, $1 \% \mathrm{w} / \mathrm{v}$; Nanjing Jiancheng Bioengineering Institute, Nanjing, China) at $37^{\circ} \mathrm{C}$. The cell suspension was transferred to four round-bottom Falcon tubes and allophycocyanin-labeled anti-cluster of differentiation (CD) 29 (cat. no. 10,225; 1:500; BioLegend, Inc., San Diego, CA, USA), fluorescein isothiocyanate-labeled anti-CD44 (cat. no. 103,003; 1:500; BioLegend, Inc.), phycoerythrin (PE) -labeled anti-CD45 (cat. no. 103,111; 1:500; BioLegend, Inc.) and PE-labeled anti-CD34 (cat. no. bsm-10820M; 1:500; BIOSS, Beijing, China) antibodies were added into the respective cell suspensions and incubated at $4^{\circ} \mathrm{C}$ for $20 \mathrm{~min}$. A flow cytometer (FACSCanto II; BD Biosciences, Franklin Lakes, NJ, USA) was used to detect the cell surface markers CD44, CD29, CD45 and CD34 (23), and Kaluza analysis software (v. 1.3; Beckman Coulter, Inc., Shanghai, China) was used for analysis. The experiment was repeated three times.

BMSC culture. Lyophilized BMP-2-nHAP-COL scaffolds were sterilized with ethylene oxide, washed three times with PBS and $\alpha \mathrm{MEM}$ and subsequently soaked in $10 \%$ FBS overnight at $37^{\circ} \mathrm{C}$. Individual scaffolds were placed into wells of a 24-well cell culture plate and inoculated with $1 \mathrm{ml} \mathrm{BMSC}$ suspension $\left(2 \times 10^{5} / \mathrm{ml}\right)$. A total of $100 \mu \mathrm{l}$ of Dulbecco's Modified Eagle's Medium (Shanghai Beinuo Biotechnology, Co., Ltd., Shanghai, China) was gently added to the well around the scaffold and the cell-seeded scaffold was cultured in a $5 \% \mathrm{CO}_{2}$ incubator at $37^{\circ} \mathrm{C}(24)$. 


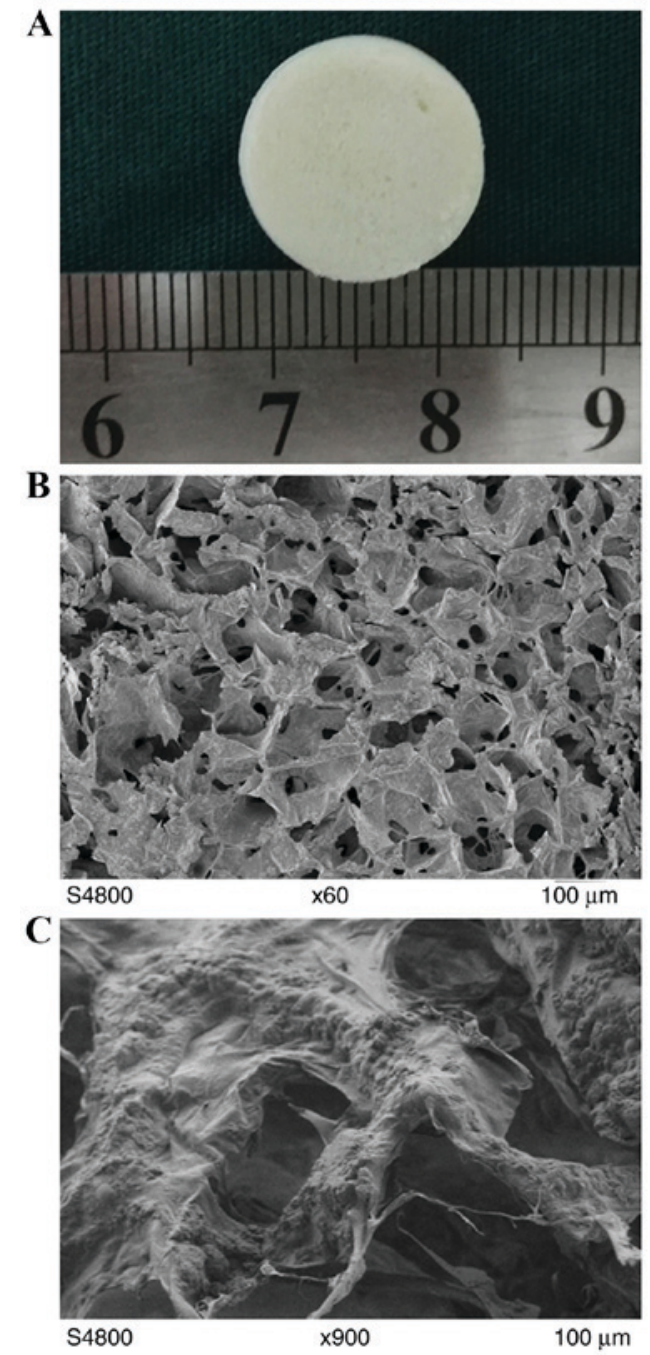

Figure 1. BMP-2-nHAP-COL scaffold characterization. (A) The gross morphology of the BMP-2-nHAP-COL scaffold was observed to be white and slightly rough on the surface. (B) Images captured of the BMP-2-nHAP-COL scaffold with a scanning electron microscope (magnification, x60) revealed a porous, spongy appearance. (C) A large number of nHAP particles attached to the scaffold surface (magnification, $\mathrm{x} 900$ ). BMP-2, bone morphogenetic protein 2 ; nHAP, nanometer hydroxyapatite; $\mathrm{COL}$, collagen.

BMSC morphology. Following $72 \mathrm{~h}$ of incubation, cell-scaffold samples were collected, washed with PBS and fixed in $2.5 \%$ glutaraldehyde solution overnight at $4^{\circ} \mathrm{C}$. The samples were subsequently removed and washed with PBS prior to dehydration in an ethanol gradient $(50,70,80,90$ and $100 \%$ for $\sim 20$ min each). Samples were air-dried and sputter-coated with gold. SEM was used to observe the samples. The experiment was repeated four times.

Adhesion of BMSCs. BMSCs $\left(1 \times 10^{4} / \mathrm{ml}\right)$ were placed in culture plates pre-coated with BMP-2-nHAP-COL or nHAP-COL scaffolds $(1 \mathrm{ml} / \mathrm{well})$. BMSCs placed in culture plates with no scaffolds were used as a control. A total of six parallel wells were used for each group and cells were cultured in an incubator at $37^{\circ} \mathrm{C}$ with $5 \% \mathrm{CO}_{2}$. Non-adherent cell numbers were quantified at 1, 2, 4, 8 and $24 \mathrm{~h}$, and the adhesion rate was calculated according to the following formula: Adhesion rate $(\%)=($ number of seeded cells-non-adhered cells)/(number of seeded cells) x100 (25). The experiment was repeated three times.
Cell Counting kit-8 (CCK-8) assay. A CCK-8 kit (Nanjing Jiancheng Bioengineering Institute) was used to detect BMSC proliferation in each group. A total of three samples per group were analyzed at 1, 3, 5 and 7 days post-inoculation. In each group, $100 \mu$ l CCK-8 solution was added to each well, and the culture plate was placed into an incubator at $37^{\circ} \mathrm{C}$ and $5 \% \mathrm{CO}_{2}$ for $4 \mathrm{~h}$. A sample of this liquid ( $300 \mu \mathrm{l})$ was subsequently drawn from each well and added to a 96-well culture plate. Absorbance values were measured at a wavelength of $450 \mathrm{~nm}$ using a microplate reader (Bio-Rad 550; Bio-Rad Laboratories, Inc., Hercules, CA, USA) and the average value of the three samples was calculated. Optical density (OD) at $450 \mathrm{~nm}$ was proportional to the number of cells (26). The experiment was repeated three times.

Alkaline phosphatase (ALP) activity. A total of three samples per group were analyzed for ALP activity at 1, 4, 7 and 10 days following initial culture using an ALP ELISA kit (cat. no. A059-1; Nanjing Jiancheng Bioengineering Institute). Samples were washed three times in PBS and immersed in $1 \mathrm{ml} 0.1 \%$ Triton X-100. Cells were lysed by placing the culture plate in a refrigerator overnight at $4^{\circ} \mathrm{C}$. The cell suspension was further lysed by repeat pipetting and $30 \mu \mathrm{l}$ suspension was subsequently transferred to a 96-well plate. Buffer solution $(50 \mu \mathrm{l})$ and matrix liquid were placed in a water bath at $37^{\circ} \mathrm{C}$ for 15 min prior to mixing fully. Chromogenic agent $(150 \mu \mathrm{l})$ was added to each well and the plate was oscillated. The OD was measured at $520 \mathrm{~nm}$ (27). The experiment was repeated three times.

Statistical analysis. All quantitative data are expressed as the mean \pm standard deviation. Statistical analyses were performed using SPSS 17 (SPSS, Inc., Chicago, IL, USA). The results were analyzed using either the Student's t-test or one-way analysis of variance (ANOVA) followed by Scheffé's post hoc test. $\mathrm{P}<0.05$ was considered to indicate a statistically significant difference. Prior to analysis with one-way ANOVA, all quantitative data were confirmed to be normally distributed.

\section{Results}

BMP-2-nHAP-COL scaffold characterization. The scaffold was white with a slightly rough surface and good flexibility. The original state was able to be gradually restored following compression deformation (Fig. 1A). The scaffold had a three-dimensional porous structure with a large number of nHAP particulates adhered on the COL surface. The aperture was $80-200 \mu \mathrm{m}$ and the pores were interconnected with no clear fixed direction. The thickness of the pore wall was 1-2 $\mu \mathrm{m}$, and nHAP and COL were well combined (Fig. 1B and C).

Release of BMP-2 from the BMP-2-nHAP-COL scaffold. BMP-2 release from the scaffold was detected for 19 days. On day 20, a negligible amount of BMP-2 ( $<1 \mathrm{ng})$ was detected in the supernatant of each sample, and the cumulative release of BMP-2 from the scaffold material was $90.05 \pm 2.08 \%$. The rate of release was significantly faster in the first few days and the release curve gradually leveled off prior to slowing (Fig. 2). 
Cell morphology. Primary BMSCs were inoculated for $12 \mathrm{~h}$ and gradually began to adhere to the scaffold. A small number of BMSCs were deformed after 1 day and were spindle- or polygonal-shaped. A number of non-adherent red blood cells were additionally observed (Fig. 3A). Non-adherent and slowly-adhering cells were removed following the first media exchange. Approximately 5 days subsequent to the initial culture, a large number of spindle and polygonal-shaped BMSCs were observed, combined with a small number of round cells. Additionally, fibroblast-like and macrophage-like cells were present (Fig. 3B). Cell impurity decreased significantly following the first passage, and cells adhered to the culture wall and deformed on day 1 (Fig. 3C). Cell density increased on day 3 following the second passage and BMSCs were approximately $80-90 \%$ confluent on day 5 (Fig. 3D).

BMSC identification. Flow cytometry for cell surface markers was performed on third-generation BMSCs. CD45, CD34, CD44 and CD29 expression levels were determined to be 4.4, 6.8, 94 and $100 \%$, respectively. These results are consistent with flow cytometry BMSC standards (CD45 and CD34, $<10 \%$; CD44 and CD29, >90\%; Fig. 4) (23).

BMP-2-nHAP-COL scaffold biocompatibility. SEM analysis identified a random distribution of cells on the scaffold surface. At 7 days, an increased number of cells had adhered to the scaffold surface and the cells were observed to grow and proliferate well. Typical BMSC morphology was observed and cells adhered tightly to the scaffold surface via lamellipodia and filopodia, indicative of cell spreading (Fig. 5).

BMP-2 promotes BMSC adhesion. The cell adhesion rate was enhanced with increased incubation time in all three groups. The adhesion rate was higher in the scaffold groups compared with the control group $(\mathrm{P}<0.05)$ and was higher in the BMP-2-nHAP-COL group compared with the nHAP-COL group $(\mathrm{P}<0.05)$ following culture for 1,3 and $6 \mathrm{~h}$. The adhered cell number was significantly increased following 3 and $6 \mathrm{~h}$ compared with $1 \mathrm{~h}(\mathrm{P}<0.05)$. These results indicated that BMP-2 increased BMSC adhesion (Fig. 6).

BMSC proliferation is not influenced by BMP-2. BMSC proliferation at days 1, 3, 5 and 7 in the BMP-2-nHAP-COL, nHAP-COL and control groups was compared using the CCK-8 assay. Absorbance values increased with time in the scaffold groups, indicating significant cell growth within the scaffolds. No significant difference was detected between the scaffold groups and the control group at day $1(\mathrm{P}>0.05)$. However, cell numbers in the scaffold groups were higher compared with the control group at day $3(\mathrm{P}<0.05)$. There was no significant difference detected between the BMP-2-nHAP-COL and nHAP-COL groups $(\mathrm{P}>0.05)$, indicating that BMP-2 did not increase BMSC proliferation (Fig. 7).

BMP-2 promotes ALP activity in BMSCs. The ALP activity of BMSCs cultured in the scaffold and control groups are presented in Fig. 8. No significant differences in the OD values were identified between the scaffold groups and the control group $(\mathrm{P}>0.05)$ during the first 4 days. However, OD values were significantly higher in the scaffold groups compared with

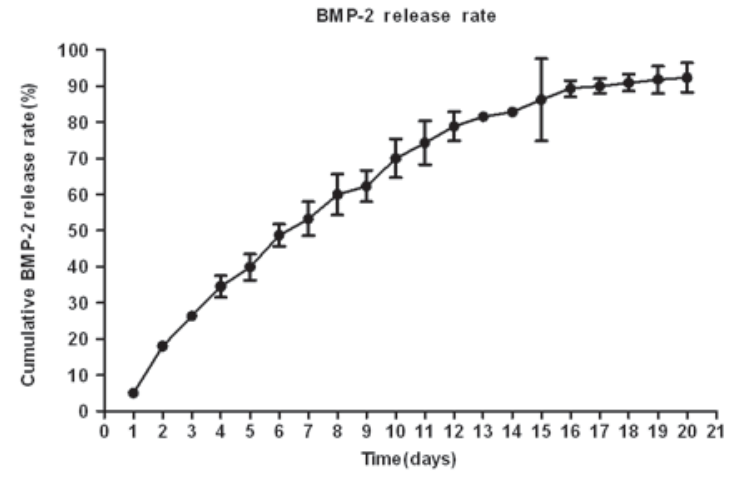

Figure 2. Cumulative release rate of BMP-2 from BMP-2-nanometer hydroxyapatite-collagen scaffolds over 20 days. BMP-2, bone morphogenetic protein 2 .

the control group between 4 and 10 days $(\mathrm{P}<0.05)$. Furthermore, ALP activity was higher in the BMP-2-nHAP-COL group compared with the nHAP-COL group, suggesting that the nHAP-COL scaffold enhanced ALP expression in BMSCs, and that BMP-2 further enhanced this effect (Fig. 8).

\section{Discussion}

COL is a primary component of the extracellular matrix that has been widely used in constructive remodeling to facilitate cell growth and differentiation. The widespread use of COL across numerous clinical applications is due to its desirable bioinductive, mechanical and degradable properties (28). In the process of constructing scaffolds and load factors, collagen was repeatedly dried, which attenuated the decrease of the immunogenicity of COL (29). HAP is a biocompatible material with osteoconductive properties. It is available in various forms that determine its bone formation and graft incorporation properties, accordingly. Unfortunately, bone-graft substitutes consisting solely of particles are mechanically weak and particles may migrate from the graft site prior to the ingrowth of new bone tissue that secures them in place (30). Nano-scale biomaterials have gained attention from the research community due to their desirable biological and biomechanical properties (31). The nHAP-COL scaffold provides a good, spongy, porous structure that meets the criteria for an ideal scaffold material, providing a three-dimensional space for cell nutrient transfer (32). The nHAP particles increase the surface roughness, thus increasing the surface area of the scaffold to improve cell adhesion. Li et al (33) used electrospinning to combine BMP-2 with silk fibroin fiber and nHAP, producing a beneficial effect on the osteogenic differentiation of BMSCs. The present study used freeze drying to directly combine BMP-2 with the nHAP-COL scaffold. The rate of BMP-2 release compared with the amount of BMP-2 in the scaffold following lyophilization revealed that the method resulted in no significant loss of BMP-2 during the production process. The sustained release time following lyophilization additionally met tissue engineering requirements. A number of disinfection methods, including alcohol immersion, ultraviolet irradiation and ethylene oxide, were used in the previous experiment. However, each method resulted in different activity loss due to different disinfection times (Tong et al, unpublished). Ethylene 
A

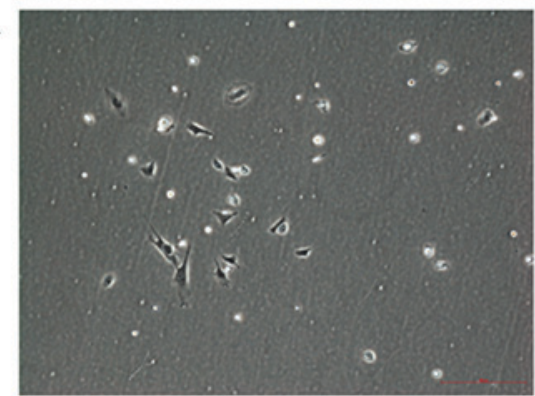

C

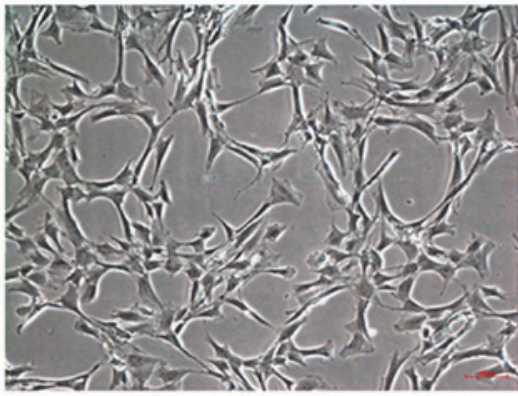

B

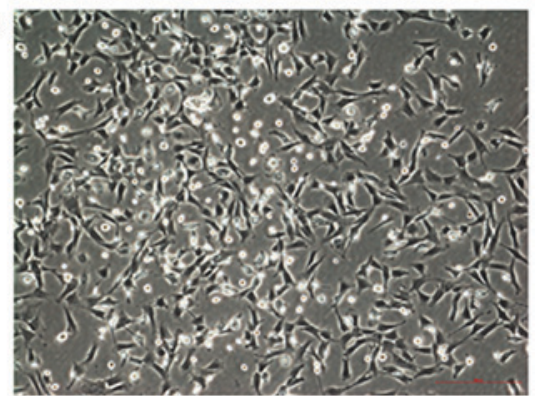

D

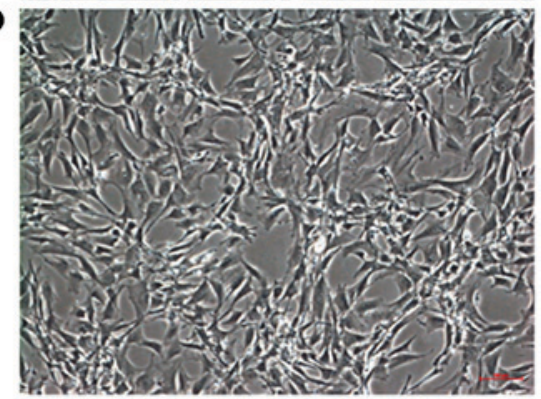

Figure 3. BMSC morphology was examined under a light-inverted microscope. (A) Cell morphology was observed following culturing of primary BMSCs for 1 day. BMSCs were mixed with non-adherent red blood cells and exhibited spindle or polygonal morphology (magnification, x100). (B) BMSCs mixed with fibroblasts and macrophages exhibited round, spindle and polygonal morphology 5 days subsequent to the primary BMSC culture (magnification, x100). (C) Cultured BMSCs grew well and cell impurity was significantly reduced 1 day following the first passage (magnification, x200). (D) The density of cultured BMSCs was significantly increased 5 days following the second passage (magnification, x200). BMSCs, bone marrow-derived mesenchymal stem cells.
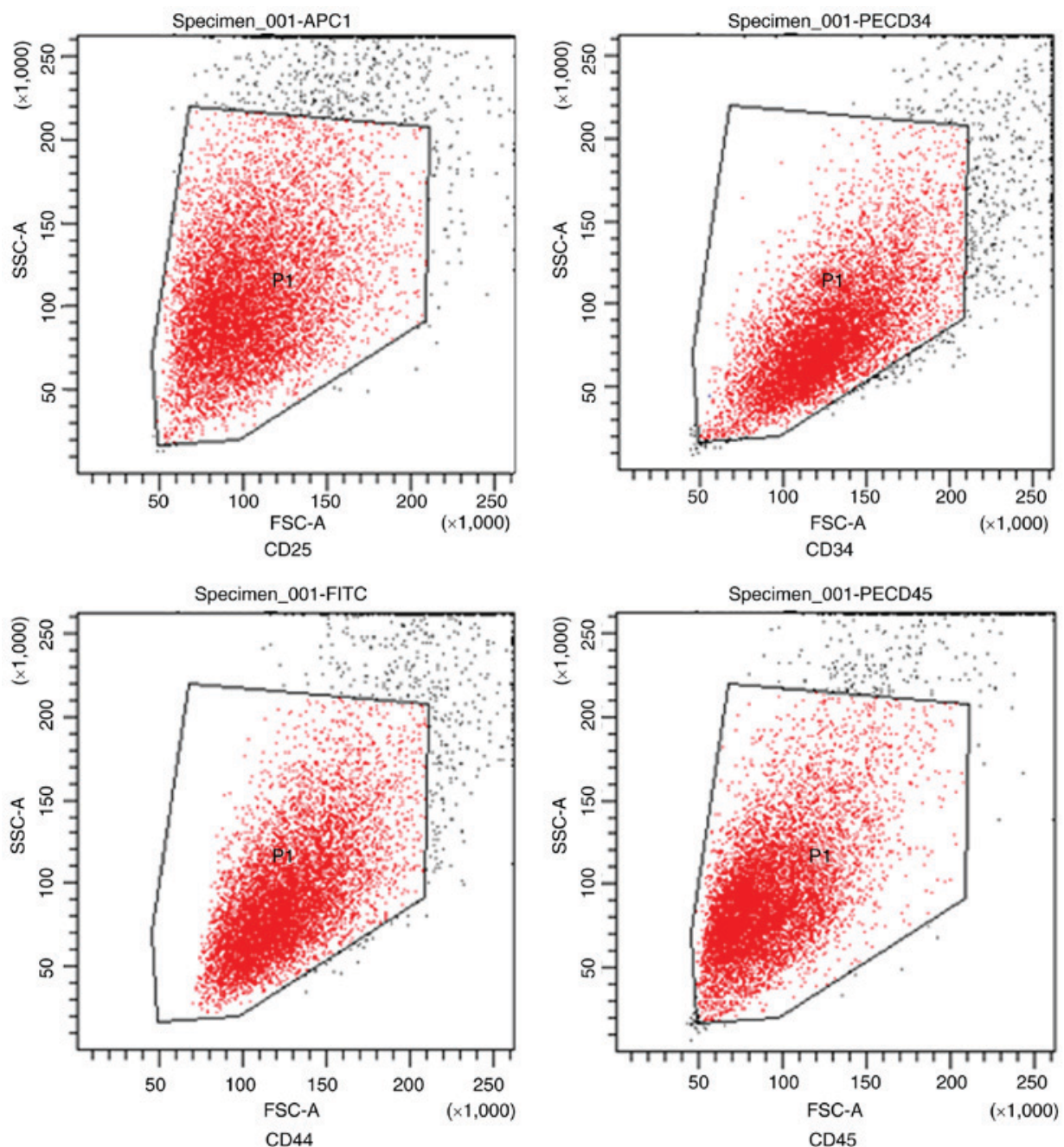

Figure 4. Flow cytometry analysis of surface marker antigens CD29, CD34, CD44 and CD45 in passage 3 bone marrow-derived mesenchymal stem cells. CD, cluster of differentiation; FITC, fluorescein isothiocyanate; PE, phycoerythrin; APC, allophycocyanin. 


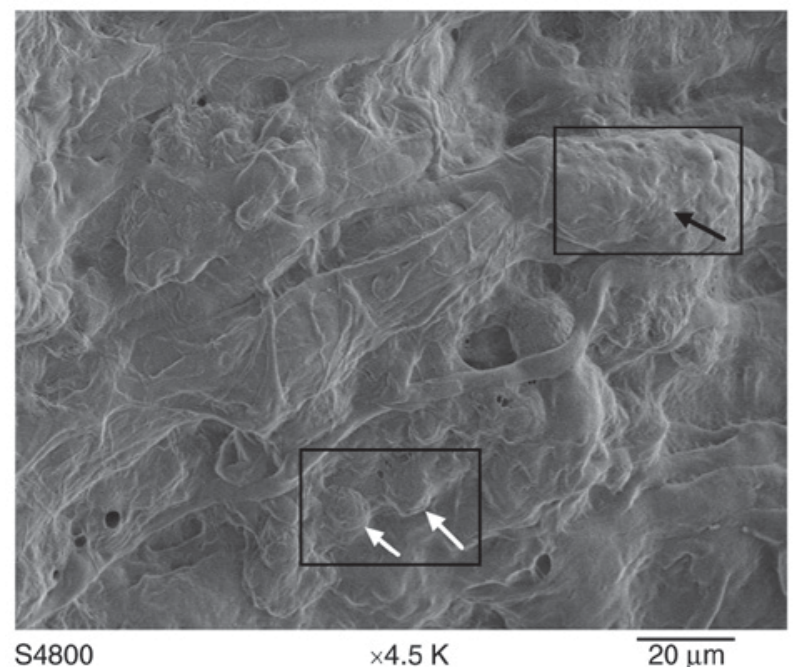

Figure 5. BMSCs at 7 days post-inoculation with the bone morphogenetic protein-2-nanometer hydroxyapatite-collagen scaffold. Images were captured with a scanning electron microscope. BMSCs adhered tightly on the scaffold surface via lamellipodia and filopodia. The white arrows point to BMSCs and the black arrow indicates the scaffold. BMSCs, bone marrow-derived mesenchymal stem cells.

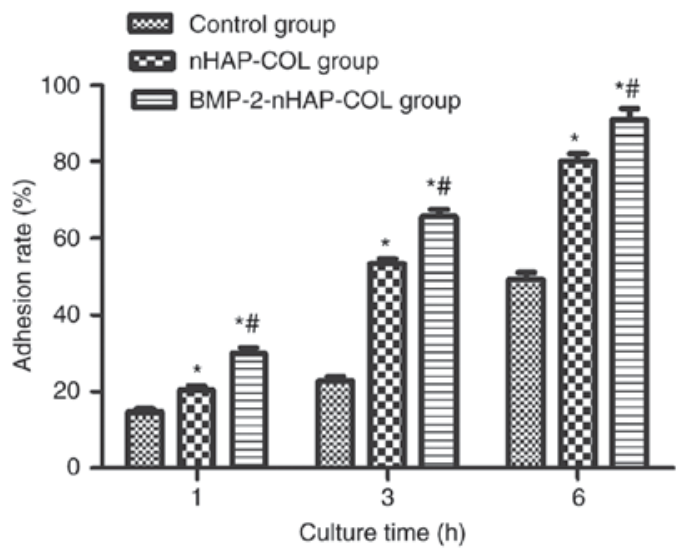

Figure 6. Adhesion rate of bone marrow mesenchymal stem cells cultured with the BMP-2-nHAP-COL and nHAP-COL scaffolds. Data is expressed as the mean \pm standard deviation. ${ }^{*} \mathrm{P}<0.05$ vs. control, ${ }^{*} \mathrm{P}<0.05$ vs. nHAP-COL group. BMP-2, bone morphogenetic protein 2 ; nHAP, nanometer hydroxyapatite; COL, collagen.

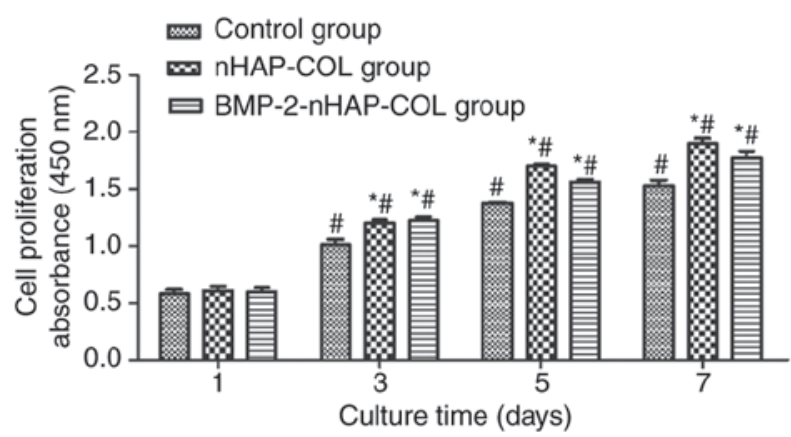

Figure 7. Cell Counting kit- 8 assay determination of the proliferation of bone marrow mesenchymal stem cells cultured with BMP-2-nHAP-COL and nHAP-COL scaffolds. Data is expressed as the mean \pm standard deviation. ${ }^{*} \mathrm{P}<0.05$ vs. control, ${ }^{\#} \mathrm{P}<0.05$ vs. previous time point of the same group BMP-2, bone morphogenetic protein 2; nHAP, nanometer hydroxyapatite; COL, collagen.

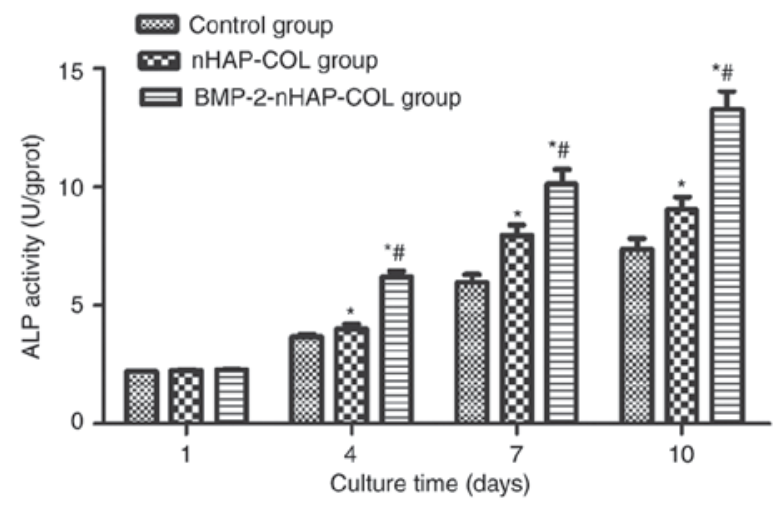

Figure 8. ALP activity assay of bone marrow mesenchymal stem cells cultured with the BMP-2-nHAP-COL and nHAP-COL scaffolds. Data is expressed as the mean \pm standard deviation. ${ }^{*} \mathrm{P}<0.05$ vs. control, ${ }^{*} \mathrm{P}<0.05$ vs. $\mathrm{nHAP}-\mathrm{COL}$ group. ALP, alkaline phosphatase; BMP-2, bone morphogenetic protein 2 ; nHAP, nanometer hydroxyapatite; COL, collagen.

oxide disinfection had the lowest rate of BMP-2 activity loss at $\sim 10 \%$, and subsequent experimental steps confirmed its rationality. No further experiments were performed to demonstrate factor concentration and specific disinfection. This may be addressed in future experiments.

Artificially-extracted BMSCs were mixed with fibroblast and macrophage-like cells. BMSCs were digested with TrypLe Express enzyme and subsequently purified. Flow cytometry analysis revealed that the isolated and purified cells met the requirements for application in further experiments. SEM images confirmed successful BMSC growth on the scaffolds. The cell adhesion rate assay confirmed that the cells adhered well to the scaffold and that BMP-2 enhanced this adherence rate. There are numerous methods of detecting cell proliferation, including CCK-8, MTT, proliferating cell nuclear antigen and Ki67. The CCK-8 assay was used in the present study. The result proved the effect of the composite scaffold on promoting proliferation. However, at a concentration of $10 \mathrm{ng} / \mu \mathrm{l}$, BMP-2 had no further role in proliferation promotion. The ALP assay confirmed that the nHAP-COL scaffold promoted BMSC differentiation and that this effect was enhanced further by the presence of BMP-2 in the scaffold. Although a number of reports have used $\mathrm{COL}$ or $\mathrm{nHAP}$ as a tissue engineering material (34), to the best of our knowledge, a combination of these two materials with BMP-2 has not yet been achieved by lyophilization. The process of freeze-drying rarely causes a loss of growth factor efficiency (35). Therefore, it may be assumed that scaffold implantation into the body may facilitate sustained BMP-2 release from the scaffold and may contribute to enhanced bone formation.

In conclusion, construction of the BMP-2-nHAP-COL scaffold using a freeze-drying method enables good biocompatibility in vitro. On this basis, further research may be performed to develop a more optimal bone tissue engineering scaffold.

\section{Acknowledgements}

The present study was supported by the Science and Technology Plan Project of Liaoning Province (grant no. 2012-B-00002012225082). 


\section{Competing interests}

The authors declare that they have no competing interests.

\section{References}

1. Zhou $\mathrm{H}$ and $\mathrm{Xu} \mathrm{HH}$ : The fast release of stem cells from alginate-fibrin microbeads in injectable scaffolds for bone tissue engineering. Biomaterials 32: 7503-7513, 2011.

2. Mao JJ, Vunjak-Novakovic G, Mikos AG and Atala A: Regenerative medicine: Translational approaches and tissue engineering. Artech House, Boston, MA, 2007.

3. Boccaccio A, Ballini A, Pappalettere C, Tullo D, Cantore S and Desiate A: Finite element method (FEM), mechanobiology and biomimetic scaffolds in bone tissue engineering. Int J Biol Sci 7: $112-132,2011$

4. Liu X and Ma PX: Polymeric scaffolds for bone tissue engineering. Ann Biomed Eng 32: 477-486, 2004.

5. Ma L, Gao C, Mao Z, Zhou J and Shen J: Biodegradability and cell-mediated contraction of porous collagen scaffolds: The effect of lysine as a novel crosslinking bridge. J Biomed Mater Res A 71: 334-342, 2004.

6. Harley BA, Leung JH, Silva EC and Gibson LJ: Mechanical characterization of collagen-glycosaminoglycan scaffolds. Acta Biomater 3: 463-474, 2007.

7. Ikada Y: Challenges in tissue engineering. J R Soc Interface 3: 589-601, 2006.

8. Appleford MR, Oh S, Oh N and Ong JL: In vivo study on hydroxyapatite scaffolds with trabecular architecture for bone repair. J Biomed Mater Res A 89: 1019-1027, 2009.

9. Karageorgiou V and Kaplan D: Porosity of 3D biomaterial scaffolds and osteogenesis. Biomaterials 26: 5474-5491, 2005.

10. King WJ and Krebsbach PH: Growth factor delivery: How surface interactions modulate release in vitro and in vivo. Adv Drug Deliv Rev 64: 1239-1256, 2012.

11. Hatakeyama W, Taira M, Chosa N, Kihara H, Ishisaki A and Kondo H: Effects of apatite particle size in two apatite/collagen composites on the osteogenic differentiation profile of osteoblastic cells. Int J Mol Med 32: 1255-1261, 2013.

12. Feng P, Niu M, Gao C, Peng S and Shuai C: A novel two-step sintering for nano-hydroxyapatite scaffolds for bone tissue engineering. Sci Rep 4: 5599, 2014.

13. He Y, Dong Y, Cui F, Chen X and Lin R: Ectopic osteogenesis and scaffold biodegradation of nano-hydroxyapatite-chitosan in a rat model. PLoS One 10: e0135366, 2015

14. Hu J, Zhou Y, Huang L, Liu J and Lu H: Effect of nano-hydroxyapatite coating on the osteoinductivity of porous biphasic calcium phosphate ceramics. BMC Musculoskelet Disord 15: 114, 2014.

15. Nukavarapu SP, Kumbar SG, Brown JL, Krogman NR, Weikel AL, Hindenlang MD, Nair LS, Allcock HR and Laurencin CT: Polyphosphazene/nano-hydroxyapatite composite microsphere scaffolds for bone tissue engineering. Biomacromolecules 9 : $1818-1825,2008$

16. Ganesh N, Ashokan A, Rajeshkannan R, Chennazhi K, Koyakutty $\mathrm{M}$ and Nair SV: Magnetic resonance functional nano-hydroxyapatite incorporated poly (caprolactone) composite scaffolds for in situ monitoring of bone tissue regeneration by MRI. Tissue Eng Part A 20: 2783-2794, 2014.

17. Vanhatupa S, Ojansivu M, Autio R, Juntunen $M$ and Miettinen S: Bone morphogenetic protein-2 induces donor-dependent osteogenic and adipogenic differentiation in human adipose stem cells. Stem Cells Transl Med 4: 1391-1402, 2015.

18. Wang YK, Yu X, Cohen DM, Wozniak MA, Yang MT, Gao L, Eyckmans J and Chen CS: Bone morphogenetic protein-2-induced signaling and osteogenesis is regulated by cell shape, RhoA/ROCK and cytoskeletal tension. Stem Cells Dev 21: 1176-1186, 2012.
19. Castro-Govea Y, Cervantes-Kardasch VH, Borrego-Soto G, Martínez-Rodríguez HG, Espinoza-Juarez M, Romero-Díaz V, Marino-Martínez IA, Robles-Zamora A, Álvarez-Lozano E, Padilla-Rivas GR et al: Human bone morphogenetic protein 2 -transduced mesenchymal stem cells improve bone regeneration in a model of mandible distraction surgery. J Craniofac Surg 23: 392-396, 2012.

20. Vilquin JT and Rosset P: Mesenchymal stem cells in bone and cartilage repair: Current status. Regen Med 1: 589-604, 2006.

21. Fujioka-Kobayashi M, Sawada K, Kobayashi E, Schaller B, Zhang Y and Miron RJ: Recombinant human bone morphogenetic protein 9 (rhBMP9) induced osteoblastic behavior on a collagen membrane compared with rhBMP2. J Periodontol 87: e101-e107, 2016.

22. Su J, Xu H, Sun J, Gong X and Zhao H: Dual delivery of BMP-2 and bFGF from a new nano-composite scaffold, loaded with vascular stents for large-size mandibular defect regeneration. Int J Mol Sci 14: 12714-12728, 2013.

23. Song K, Huang M, Shi Q, Du T and Cao Y: Cultivation and identification of rat bone marrow-derived mesenchymal stem cells Mol Med Rep 10: 755-760, 2014.

24. Tong S, Xue L, Xu DP, Liu ZM, Du Y and Wang XK: In vitro culture of hFOB1.19 osteoblast cells on TGF- $\beta 1$-SF-CS three dimensional scaffolds. Mol Med Rep 13: 181-187, 2016.

25. Wang Z, Lin M, Xie Q, Sun H, Huang Y, Zhang D, Yu Z, Bi X, Chen J, Wang J, et al: Electrospun silk fibroin/poly (lactide-co- $\varepsilon$-caprolactone) nanofibrous scaffolds for bone regeneration. Int J NanoMedicine 11: 1483-1500, 2016.

26. Zhang H, Ma X, Zhang L, Guan X, Bai T and Xue C: The ability to form cartilage of NPMSC and BMSC in SD rats. Int J Clin Exp Med 8: 4989-4996, 2015.

27. Gao P, Zhang H, Liu Y, Fan B, Li X, Xiao X, Lan P, Li M, Geng L, Liu D, et al: Beta-tricalcium phosphate granules improve osteogenesis in vitro and establish innovative osteo-regenerators for bone tissue engineering in vivo. Sci Rep 6: 23367, 2016.

28. Chan EC, Kuo SM, Kong AM, Morrison WA, Dusting GJ, Mitchell GM, Lim SY and Liu GS: Three Dimensional Collagen Scaffold Promotes Intrinsic Vascularisation for Tissue Engineering Applications. PLoS One 11: e0149799, 2016.

29. Xu C, Lu W, Bian S, Liang J, Fan Y and Zhang X: Porous collagen scaffold reinforced with surfaced activated PLLA nanoparticles. Scientific World Journal 2012: 695137, 2012.

30. Lee MJ, Sohn SK, Kim KT, Kim CH, Ahn HB, Rho MS, Jeong MH and Sun SK: Effect of hydroxyapatite on bone integration in a rabbit tibial defect model. Clin Orthop Surg 2: 90-97, 2010.

31. Xia Y, Zhou P, Cheng X, Xie Y, Liang C, Li C and Xu S: Selective laser sintering fabrication of nano-hydroxyapatite/poly- $\varepsilon$-caprolactone scaffolds for bone tissue engineering applications. Int J Nanomedicine 8: 4197-4213, 2013.

32. Zeng S, Liu L, Shi Y, Qiu J, Fang W, Rong M, Guo Z and Gao W: Characterization of silk fibroin/chitosan $3 \mathrm{~d}$ porous scaffold and in vitro cytology. PLoS One 10: e0128658, 2015.

33. Li C, Vepari C, Jin HJ, Kim HJ and Kaplan DL: Electrospun silk-BMP-2 scaffolds for bone tissue engineering. Biomaterials 27 : 3115-3124, 2006.

34. Polo-Corrales L, Latorre-Esteves $M$ and Ramirez-Vick JE: Scaffold design for bone regeneration. J Nanosci Nanotechnol 14: 15-56, 2014.

35. Wang W: Lyophilization and development of solid protein pharmaceuticals. Int J Pharm 203: 1-60, 2000.

This work is licensed under a Creative Commons Attribution-NonCommercial-NoDerivatives 4.0 International (CC BY-NC-ND 4.0) License. 